\title{
Exploring the experiences of Iranian adolescent mothers about the maternal role: a qualitative study
}

\author{
Maasoumeh Mangeli ${ }^{1}$, Batool Tirgari ${ }^{2}$, Mohammad Ali Cheraghi ${ }^{3}$, Masoud Rayyani ${ }^{1}$
}

${ }^{1}$ Ph.D. of Nursing, Assistant Professor. Nursing Research Center, Razi School of Nursing and Midwifery, Kerman University of Medical Sciences, Kerman, Iran

${ }^{2}$ Ph.D. of Nursing, Associate Professor, Nursing Research Center, Razi School of Nursing and Midwifery, Kerman University of Medical Sciences, Kerman, Iran

${ }^{3}$ Ph.D. of Nursing, Full Professor, School of Nursing and Midwifery, Tehran University of Medical Sciences, Tehran, Iran

\section{Type of article: Original}

\begin{abstract}
Background: Early motherhood is one of the most important issues in developing countries. Adolescent mothers are not sufficiently prepared to accept the maternal role. How to achieve success in the maternal role should be studied.

Objective: To explore the experiences of Iranian adolescent mothers about the maternal role.

Methods: This qualitative study was conducted through a conventional content analysis approach from March to December 2016. Face to face in-depth semi-structured interviews were conducted with 18 Iranian teenage mothers in Kerman province, Iran. Participants were selected purposefully and with maximum variation of the age of the first birth, child's age, place of residence and financial status. Data collection continued until data saturation and MAXQDA software was utilized to analyze the collected data.

Results: Data analysis resulted in the extraction of the two main categories including optimizing the process of the maternal experience (use of maximum individual capabilities, planning and management of conditions, efforts to acquire maternal competency, and reliance on spirituality) and supporters for the acceptance of the maternal role (social support, professional services of health care providers, time lapse, care history).

Conclusion: Early motherhood is not merely an unpleasant experience; if appropriate strategies and facilitators are used, it can have positive consequences. Health care providers should provide satisfactory services in care, education and support with adequate knowledge of adolescent characteristics. Opportunities in the community should also be directed to support this vulnerable group.
\end{abstract}

Keywords: Maternal, Role, Adolescent, Qualitative Research, Iran

\section{Introduction}

Becoming a mother is the most crucial event for women through which a period of change, instability and reorganization of life happens (1). Maternal role attainment is a process in which the mother attains required competences for the maternal role, learns appropriate behaviors, establishes herself in the role, and accepts maternal identity (2). This process can be influenced by the individuals' characteristics, outlooks and beliefs, social and economic condition, preparedness, knowledge and skills, and psychological status (1). Success in the maternal role comes when the mother acquires competence for care of the child, loves her child, is committed to him, accepts maternal responsibilities, and creates balance between the new role and previous roles (3). The maternal outcomes of success in the maternal role include confidence, gratification in the role, and attachment to the child. In addition, a child's outcomes are cognitive and emotional development, attachment, health, and social competence (2). Although

\section{Corresponding author:}

Assistant Professor Dr. Masoud Rayyani, Nursing Research Center, Razi School of Nursing and Midwifery, Kerman University of Medical Sciences, Kerman, Iran.

Tel: +98.3431325219, Fax:+98.3431325218, Email: m_rayani@kmu.ac.ir

Received: August 30, 2017, Accepted: October 28, 2017, Published: May 2018

iThenticate screening: October 16, 2017, English editing: April12, 2018, Quality control: April 15, 2018

This article has been reviewed / commented by six experts

(C) 2018 The Authors. This is an open access article under the terms of the Creative Commons Attribution-NonCommercialNoDerivs License, which permits use and distribution in any medium, provided the original work is properly cited, the use is non-commercial and no modifications or adaptations are made. 
maternal preparedness is usually raised in adulthood, the increasing number of teenage mothers is one of the important concerns in many countries (4). According to the World Health Organization, every year, approximately 16 million teenage girls give birth worldwide, while South Korea designates the minimum rate, and the maximum rate goes for sub-Saharan Africa (5). Motherhood in adolescence is very important because adolescent mothers must simultaneously overcome two developmental crises of motherhood and adulthood (3). Early motherhood is often considered both problematic and undesirable (6). Teen mothers face many challenges related to themselves, family, school, workplace, and community (7). These challenges can be the constant need for support and training (4), inability to plan ahead or make decisions, lack of maternal skills (8), encountering unknown situations and major changes (4), high risk pregnancy and birth (9), mental health problems (depression, anxiety, shock, low selfefficacy, isolation) (10), multiple responsibilities (11), role conflict and identity confusion (12), inadequate social support (7), disruption of education and employment (11), financial problems, social stigma (8), and family conflicts $(9,13)$. Teenage mothers must be able to cope with the challenges. According to the results of studies, teenage mothers use several strategies for success in the maternal role these can be in the form of resilience $(14,15)$, reinforcement of adaptive power, positive thinking, self-efficacy (16), restarting and establishing supportive relationships (17), and technology-based instruction (18-20). In addition, researchers have shown that health services $(12,21,22)$, social support $(3,10,12)$, financial support, removal of stigma, and cultural support (23) helps teenage mothers to success in the maternal role. Overcoming challenges leads to positive educational, career and parenting outcomes for teenage mothers (24). Cultural conditions and social support play an important role in the success of adolescent mothers in the maternal role (3). According to the last census in Iran, the highest number of recorded marriages was devoted to women who were 15-19 years of age. Southeastern regions of Iran such as Kerman province have a high rate of early marriage (25). Based on the Government Strategy Plan, it is expected that the fertility rate among Iranian teenage mothers will increase significantly by 2025 (5). Although in many societies, teenage mothers face social stigma, in the Islamic culture of Iran, adolescent mothers enjoy familial and social support. Iranian families support adolescent mothers in the maternal role and attempt to reduce their problems. Iranian culture emphasizes the increase in fertility rates, and mothers have an invaluable position in this culture. So, adolescent pregnancy is confirmed by Iranian culture. Most qualitative research in Iran has focused on teenage pregnancy $(5,26)$. No comprehensive qualitative research has so far been carried out on success in motherhood among Iranian adolescent mothers. Experiences of teenage mothers about their maternal role should be explored among various cultures. Discovery of the unknown and valuable aspects of accepting the maternal role in adolescents is possible only through qualitative studies. To help teenage mothers, health policymakers should recognize strategies and facilitators of success in the maternal role and make appropriate decisions in this regard. The first author of this article has experienced almost 20 years of educational and clinical care in maternal and child health, and has completed more than 10 research projects in this field. From authors' opinions, more qualitative studies are needed among Iranian teenage mothers, because this phenomenon must be explored through the study of experiences. The purpose of this study was to explore the experiences of Iranian teen mothers about the maternal role.

\section{Material and Methods}

\subsection{Research design and participants}

This was a conventional content analysis study and was conducted in 2016 in Kerman, Iran. A total of 18 teenage mothers who referred to health centers for maternity and child care and met the inclusion criteria (being a maximum of 19 years of age at the time of first birth, having a child or children up to 2 years of age, being able to speak Persian, being willing to share personal experiences, living with spouses, not having a history of severe mental illness, and good cooperation with the researcher) participated in this study. Participants were selected purposefully with maximum variation in age, child's age (3-24 months), and place of residence (urban or rural) as well as financial situation. In this study, the participants were 18 adolescents, within the ages of 14 to 18.5 at the time of childbirth. All of them were housewives and had education levels from middle school to university diploma at the time of interview. Among most cases their husbands were at least 10 years older.

\subsection{Data collection}

The data were collected through face to face in-depth semi-structured interviews conducted by the first author (i.e. a $\mathrm{PhD}$ candidate of nursing). The interviews focused on the experiences of the participants. The teenagers were asked to explain how they experienced their maternal role in adolescence, how they dealt with the challenges, what strategies they used to succeed in the maternal role and what people or organizations helped them. General questions were asked and progression was made into specifics, also in accordance with participants' statements, additional probing questions were used. The interviews were conducted at specific times and locations and lasted for, in 
agreement with the participants, 45 to 80 minutes, during a 6-month period from March to August 2016. The whole interviews were recorded and transferred into audio files to be entered into the computer and the data collection continued until data saturation was reached, i.e. when no new information was obtained from the interviews.

\subsection{Data Analysis}

The conventional content analysis approach (Graneheim \& Lundeman) was utilized for data analysis (27). The initial audio files of interviews were listened to, and recorded interviews there-after were immediately transcribed verbatim and then read several times to gain a general impression from data. The resulting text from the interviews was read line - by - line and meaningful units (words or sentence or paragraphs) were identified, which were then condensed, abstracted, coded, and labeled. Next, the codes were re-read in order to be arranged into categories and sub-categories based on their similarities and differences. The first author performed data coding and all co-authors supervised the coding process. In cases where there was disagreement over the coding, the authors discussed and negotiated the codes until they reached a compromise. Data analysis was done continuously and simultaneously with data collection wherefore the data and the generated codes were constantly compared. MAXQDA10 software was also run to record the data and categories. Data analysis lasted from March to December 2016.

\subsection{Trustworthiness}

Guba and Lincoln's criteria for determining the trustworthiness of qualitative research were utilized in this study. This criteria include credibility, transferability, dependability, and confirmability (28). In this study, credibility increased through prolonged engagement with the research, spending sufficient time for collection and analysis of data (in total 11 months), appropriate communication with participants, member check and peer check. For member check, researchers checked results of the study with numbers of participants. For peer check, all of the research process, particularly analysis of the data, was done through collaboration of the research team. In order to ensure transferability, the research process was accurately recorded to make the follow-up possible. To increase dependability, two adolescent mothers who were external to the study read the excerpts from the interviews and the generated codes, and approved that the data and the findings were almost the same as their own experiences. Also, the opinion of a reproductive health doctorate (an expert outside the research team) was used. To increase confirmability, triangulation (interview and field note) was used for gathering the data. The data analysis was repeated after any interview and the data and the generated codes were constantly compared. Also, documenting the steps of data collection and analysis was used.

\subsection{Ethical considerations}

Kerman University of Medical Sciences Human Research Committee approved this study (ethics approval number: IR.KMU.REC.1394.591). Its purpose, publishing, and implications of the results obtained were explained to the participants. The anonymity of participants, privacy and confidentiality were maintained. Interviews were conducted in a private environment and audio files were kept anonymously. Furthermore, participants were assured of the confidentiality of their responses. Participants were also informed that participation in this study was voluntary and they could withdraw at any time. Written consent forms were obtained from the participants for the recording of the interviews.

\section{Results}

Two main categories (Optimizing the process of the maternal experience, Supporters for the acceptance of maternal role) including eight sub-categories were extracted from the data analysis (Table1).

Table 1. Main categories and sub categories of factors that are successful in the maternal role among adolescent mothers

\begin{tabular}{|l|l|}
\hline Categories & Sub categories \\
\hline Optimizing the process of the maternal experience & Use of maximum individual capabilities \\
\cline { 2 - 2 } & Planning and management of conditions \\
\cline { 2 - 2 } & Efforts to acquire maternal competency \\
\cline { 2 - 2 } & Reliance on spirituality \\
\hline \multirow{5}{*}{ Supporters for the acceptance of the maternal role } & Social support \\
\cline { 2 - 2 } & Professional services of health care providers \\
\cline { 2 - 2 } & Time lapse \\
\cline { 2 - 2 } & Care history \\
\hline
\end{tabular}




\subsection{Optimizing the process of the maternal experience}

Adolescent mothers try to make the maternal experience pleasant with multiple strategies. The use of maximum individual capabilities, planning and management of conditions, efforts to acquire maternal competency, and reliance on spiritual support were the four subcategories of this category.

\subsubsection{Use of maximum individual capabilities}

Some teen mothers have been using their maximum capabilities. Optimistic acceptance of challenges, positive attitude, compliance with conditions, acceptance of the responsibility for child care and upbringing, sensitivity to the needs of the child, and provision of a safe and healthy environment for the child were examples of these efforts. Striving to change their attitudes, they tried to strengthen their sense of well-being, accept the facts, endure the misfortunes, and accept the change. An adolescent who was 14 years old and had a three-month- old infant stated: "..., I did all my baby's work. I wanted to get used to it. When my son was born, I quickly got up from my bed, I breastfed him. I instantly embraced him, and I wanted to take responsibility for him... ". Adolescent mothers who were self-confident were more likely to use their abilities, and tried to remove barriers. High self-esteem strengthened self-confidence in abilities in teenage mothers. Also, mothers who were interested in children and motherhood, overcame adversities and had a better chance of success. A 15-year-old adolescent who had a 14 month-old child and experienced a difficult pregnancy and birth, said: “...The doctor told me, pregnancy would be dangerous for me, that my labor may be very difficult. I said I was strong, I could cope. I was not worried because I wanted a child. Nobody believed that I could undergo a vaginal delivery, but I could..."

\subsubsection{Planning and management of conditions}

Adolescent mothers attempted to manage the living conditions in the face of housekeeping, taking care of the spouse, and the child-care responsibilities. Planning, managing time, using collaborators, managing cost and income, and prioritizing needs were done. With these interventions, the teenage mothers enjoyed motherhood and presence with their spouse and child, and they also enjoyed their interests. An adolescent who was 19 years old, had a 23month-old child and had been university educated said: “...In order to be able to continue my education, I chose to stay on the university campus. I took my courses so that I could tend to the baby. When my baby was sleeping, I studied or did my favorite things..."

\subsubsection{Efforts to acquire maternal competency}

Adolescent mothers who did not have enough knowledge and skill to accept the maternal role, tried to attain competency for maternity. For this purpose they studied books, participated in childbirth and breastfeeding classes, consulted experts and the family, surfed the Internet, practiced the maternal role, and worked at a kindergarten. An 18.5-year-old adolescent who had an 18-month-old child stated: “... I often found the answer to my questions on the Internet. I got help from physicians and other mothers in an online group. I explained my experience of pregnancy and birth to other members..."

\subsubsection{Reliance on spiritual support}

Relying on spirituality was another strategy of adolescent mothers to overcome problems. They considered care of children to be worthy of reward. They considered childbirth to be sacred. They considered the bitter and sweet events of life to be God's expediency. Therefore, they trusted in God, endured the afflictions and thanked God. They believed in God's kindness and hoped for divine reward. Reviewing hadiths and anecdotes, reading prayers, and attending spiritual environments increased the ability to confront problems. An adolescent who had an eight-monthold infant and considered success as the result of relying on God, said: "...I said to myself, "I put my life in the hands of God." I did not know anything about life. God placed me in the right direction. I was hoping for God when I became a mother. I believed that God would pay attention to me..."

\subsection{Supporters for accept maternal role}

This category expresses facilitators of accepting a maternal role in adolescent mothers and is made up of: social support, professional services of health care providers, time lapse, and care history. This category and its subcategories show the factors that help teenage mothers to succeed in the maternal role. This category describes situations which are appropriate for teenage mothers.

\subsubsection{Social support}

Social support was one of the most important help factors to adolescent mothers. According to participants, social support was provided in various aspects including financial, emotional, care, educational, legal and political support. Support resources included family members, neighbors, friends, school staff, law enforcement agencies, health policymakers, and government agencies. The most effective and widespread support was provided by the mother and the spouse. Mothers of teen mothers provided care, educational, emotional and financial support. Wives have also supported adolescent mothers by reducing feelings of sadness and loneliness, participation in housekeeping and child care, accompanying in health care, mental affiliation, tolerating mood and behavioral disorder, providing a 
pleasant environment, strengthening self-esteem and confidence. An adolescent who had become a mother at 15 years old stated:“...If our mother did not support us, we would face a lot of problems. I do not have enough knowledge about child care. My mother is very supportive. So we do not encounter the problems in raising a child..." The school team supported adolescent mothers by allowing them to attend school, reducing their problems and worries. The educational team of the school compensated educational disruption of the teenage mothers. They also provided consultation services for teenage mothers who had experienced stress. Friends of adolescent mothers, in addition to providing help for child care, emotionally supported them and facilitated the education of the teenage mothers. Some organizations also facilitated the acceptance of the maternal role to teenage mothers by providing legal support for them. The Ministry of Education legally supported adolescent mothers, facilitating their presence in schools. Some centers also provided financial support for teenage mothers who had financial poverty, because early marriage and motherhood had prevented them from education, skill training, and employment. Health policymakers attempted to solve the problems of adolescent mothers by creating positive attitudes in health care providers, reducing health and medical expenses, providing expert staff to provide special services, promoting home visits, providing family-based services and a specific care plan.

\subsubsection{Professional services of health care providers}

Health care providers helped teenage mothers by caring according to their needs, meeting learning needs, providing emotional support, attention to adolescents' characteristics, compassionate behavior, and devoting a sufficient amount of time and energy. Health care providers supported adolescent mothers in pre-pregnancy counseling; prenatal care, childbirth, and in postpartum; family planning, breast-feeding, and child-rearing. An adolescent who had a four-month-old infant said: "...When I went to the health center, they controlled my weight and blood pressure. They assessed the fetus situation. I was sure that the baby was healthy. They answered my questions. They addressed my worries..."

\subsubsection{Time lapse}

The passage of time enabled teen mothers to learn the skills needed to take care of the child. Over time, adolescent mothers were skilled at breastfeeding, empowered in planning, became accustomed to new situations, understood the needs of the child, their anxiety and worries had diminished, and had opportunities for their own interests. A 19year-old adolescent, 14 months after the child's birth said: “...Now, from his cries I understand what he (child) wants. I understand him now. I know what I should do. I learned a lot over this period. The passage of time has helped me to learn a lot of things through experience..."

\subsubsection{Care history}

Adolescent mothers who had a history of observing or taking care of the child were more successful in the maternal role. Also, due to parents' loss and financial problems, teenagers living in difficult conditions and enduring multiple responsibilities were more successful in maternity. An 18-year-old adolescent, who had learned from her mother how to care for her child, said: "...I had already seen how my mother cared for my brother, how she bathed him, how she fed him, how she calmed him; so I knew a lot of things..."

\section{Discussion}

This study was aimed at exploring the experiences of Iranian adolescent mothers adapting to the maternal role. Findings of this study showed that adolescent mothers use several strategies to succeed in the maternal role. Optimizing the process of the maternal experience, and supporters for the acceptance of the maternal role were the main strategies and facilitators for the success of adolescent mothers in the maternal role. One of the most important strategies that had been utilized by Iranian teenage mothers was "Use of maximum individual capabilities". Participants stated that they attempted to use all of their abilities. This strategy is often known as resilience, and has been reported in many studies $(6,14,15)$. Resilience means maintaining and enhancing capabilities despite challenges (6). This process helps the person to overcome problems. Self-efficacy, environmental mastery, internal control, and good relationships with others are very influential on resilience (14). Positive attitude, acceptance of change, ethical investment, self-empowerment and self-esteem help the adolescent mothers to build and maintain resilience (15). A 2016 study by Lévesque showed that establishing maternal identity, bonding with the child as a turning point, taking an active position in lieu of victimization, and acting proactively and adjusting to motherhood are main themes of resilience in adolescent mothers. He postulated that positive adaptation, promotion processes, and risk-taking, complete resilience (14). In another study, resilience in adolescent mothers is described with a critical review of one's own needs and environment, change to the environment to meet the needs as well as the transition of dependence on accountability. Also, self-esteem, positive thinking, adaptation to change, reasoning, and coping with peer pressure, grief, loneliness, rejection, stress and trauma are included in resilience (6). 
Planning and management of conditions were other strategies of teenage mothers for success in the maternal role. Unlike other mothers, adolescents were faced with several roles such as mothering, adolescence, and being a student. So performing the multiple roles simultaneously, required careful planning. Motherhood motivated teen mothers to review their plans. A 2015 study by Mantovani showed that many teenage mothers adapted to the new situation through proper planning, and succeeded in their educational, occupational and parenting skills (15). Planning for optimal relationships with others, access to income sources and cost management can strengthen the ability of adolescent mothers for adaptation (24). Also, goal setting, problem solving, appropriate decision making, career planning and time management can help the young mother manage new situations (14).

Efforts to acquire maternal competency, was another strategy that had been used by teenage mothers. Teenage mothers were dependent on families and health care providers, because they did not have competency in the maternal role. Most of the teenage mothers had chosen empowerment in the maternal role as the most important of priorities. They used several methods to acquire knowledge and skills, but they preferred virtual space and computer technology because of their interest. The success of this approach as a strategy - adopted by adolescent mothers and health care providers - has been endorsed in numerous studies (18-20).

The bulk of teenage mothers experienced a positive motherhood by relying on spirituality. Spirituality helped them to overcome challenges and gain extra energy to face problems. A qualitative research studied the effect of spirituality on postpartum depression among African-American women and reported that its themes consist of stress relief, feeling of value, reduction of loneliness, experiencing thanksgiving, accepting God's destiny, expanding relationships and preventing self-harm (29). Another researcher stated that faith in God strengthens the power of adaptation with the new role in mothers. She showed that worship, reading the Holy Bible, and attending spiritual sites, and religious services can help mothers adjust to their new role (30). In addition to the strategies above, the process of becoming a mother is facilitated in adolescent mothers by the professional support of health care providers, social support, time lapse, and care history. Adolescent mothers were vulnerable and needed care, and educational, emotional and social support from health care providers. Participants acknowledged that if they received sufficient support from health care providers, they could succeed in the maternal role. A 2015 study by Logsdon showed that the health literacy of teenage mothers is low, and some do not understand the usual training or do not take it seriously. They stated that educational support promotes the knowledge of adolescent mothers in breastfeeding, child care, attachment with the child, and the prevention of postpartum depression (31). In addition, the coping ability of adolescent mothers in psychological problems can be increased through training of stress management, coping strategies, and parenting skills (22). If health care providers understand the experiences and feelings of adolescent mothers, they can provide emotional support for them (21).

Another important facilitator for adolescent mothers was social support. They overcame most of the challenges, if they received support from family and community. The significance of social support for success in the maternal role of adolescent mothers has been confirmed in several studies $(3,10,32,33)$. Social support includes receiving love, care, help and support from others (32). Teen mothers are mentally and emotionally unprepared to take maternal responsibility, and need support and guidance for learning parental skills (33). Adolescent mothers need social support in child care and development, adjustment to the baby's sleep patterns, and postpartum emotional and physical changes. In addition, under certain conditions, some adolescent mothers need social support for food, clothing, housing, travel and other financial matters (10). Social support is essential for the upbringing of a child by its teenage mother, and helps them to adapt to the new role. Social support reduces stressors and improves the health of adolescent mothers (32). Family, community, organizations and cultural support can help teenage mothers care for themselves and their children and continue their education (34). Furthermore, it leads to empowerment, maternal satisfaction, and desirable parenting outcomes (3). Although in many countries, being a teenage mother is a social stigma, Iranian culture (particularly Kerman province) confirms early motherhood. In the Islamic culture of Iran, teenage pregnancy is often a result of legal marriage, and adolescent mothers enjoy familial and social support. In addition, mothers have an invaluable position in Iranian culture. A 2014 study by Schrag showed that the most important sources of family support for teen mothers are the spouse and mother. They provide love and safety for teenage mothers and enhance self-esteem, increase satisfaction, reduce stress and depression, improve knowledge and attitudes and instruct maternal skills (32). Iranian adolescent mothers, also receive significant support from families, particularly their mothers. Time lapse, and care history were other facilitators for accepting the maternal role in Iranian teenage mothers. When teenagers became mothers, they did not have enough skills, but they would gain competency with time and experience. Also, teenagers who had history of child care or observed it, were more successful in the maternal role. A 2013 study by Sriyasak showed that having the experience of caring for family 
and friends is very effective for teenage mothers (23). According to these results, teenage mothers will be successful in the maternal role if they use appropriate strategies and have access to facilitators. In dealing with teenage mothers, health care providers should consider their characteristics and abilities. They should use strength based care and emphasize the strengths of adolescent mothers. Principles of this care are collaboration, respect for individuals, empowerment, and self-direction. The foundation of this kind of care pertains to the belief that individuals have unique talents, skills, needs, and life experiences that must be valued and strengthened (22). For the improvement of consequences of teenage motherhood, health care providers should comprehensively investigate the socioeconomic and cultural conditions of adolescent mothers (35). They should use available opportunities to strengthen familial and social support for teenage mothers and their children. Healthcare providers should identify adolescent mothers' needs and implement professional programs to enhance mothers' childbearing and childrearing knowledge and skills and thus improve acceptation of the maternal role. Regarding the limitation of the study we should say that the sample size of this study was small and looked at only part of multiple cultures of Iran, so researchers suggest the need for similar research in the field.

\section{Conclusions}

This study explored experiences of Iranian teenage mothers regarding their maternal role. The findings of this study showed that teenage mothers are vulnerable, but have special abilities. They require appropriate strategies and facilitators to succeed in the maternal role. To be considered teenage mothers, health care providers need to be aware of adolescents' characteristics, and establish an intimate and respectful relationship with them. In order to help the success of adolescent mothers in the maternal role, they need to be considered and their abilities to be strengthened. In addition, environmental facilitators such as social support and professional health support should be used. Health care providers should identify the care, educational and support needs of adolescent mothers, and take appropriate interventions. Health care providers should help teenage mothers to accept responsibilities, overcome financial challenges, and actively play the maternal role by appropriate planning. Health care providers need to have a careful evaluation of the status of teenage mothers and their children at the stages of the child's development and provide appropriate services. Health care providers should also identify sources of support in the community, especially the family, and guide them to help teenage mothers. Understanding strategies and facilitators of success in a maternal role, in addition to helping adolescent mothers, can also be useful in decision making of health policymakers.

\section{Acknowledgments:}

Authors wish to thank all the adolescents who participated in this study. They also appreciate the assistance of the Ethics and Research Boards of Kerman University of Medical Sciences. This work was the production of a PhD dissertation.

\section{Conflict of Interest:}

There is no conflict of interest to be declared.

\section{Authors' contributions:}

All authors contributed to this project and article equally. All authors read and approved the final manuscript.

\section{References:}

1) Javadifar N, Majlesi F, Nikbakht A, Nedjat S, Montazeri A. Journey to Motherhood in the First Year After Child Birth. Journal of Family and Reproductive Health. 2016; 10(3): 146-53. PMID: 28101116.

2) Alligood MR. Nursing Theory Utilization and Application. 5 ed. St.Louis: ELSEVIER.MOSBY; 2014.

3) Brown SG, Hudson DB, Campbell-Grossman C, Kupzyk KA, Yates BC, Hanna KM. Social Support, Parenting Competence, and Parenting Satisfaction Among Adolescent, African American, Mothers. West J Nurs Res. 2018; 40(4): 502-19. doi: 10.1177/0193945916682724. PMID: 28322635.

4) Pogoy AM, Verzosa R, Coming NS, Agustino RG. Lived Experiences Of Early Pregnancy Among Teenagers: A Phenomenological Study. European Scientific Journal. 2014; 10(2): 157-69.

5) Mohammadi N, Montazeri S, Ardabili HE, Gharacheh M. Iranian pregnant teenage women tell the story of "fast development": A phenomenological study. Women Birth. 2015; 29(4): 303-9. doi: 10.1016/j.wombi.2015.11.003. PMID: 26651284.

6) Clarke J. It's not all doom and gloom for teenage mothers-exploring the factors that contribute to positive outcomes. International Journal of Adolescence and Youth. 2015; 20(4): 470-84. doi: $10.1080 / 02673843.2013 .804424$. 
7) Williamson JA, McCabe JE, O'Hara MW, Hart KJ, LaPlante DP, King S. Parenting stress in early motherhood: stress spillover and social support. Comprehensive Psychology. 2013; 2(11): 1-14. doi: 10.2466/10.21.CP.2.11.

8) van Zyl L, van Der Merwe M, Chigeza S. Adolescents' lived experiences of their pregnancy and parenting in a semi-rural community in the Western Cape. Social Work. 2015; 51(2): 151-73. doi: 10.15270/51-2439.

9) Vincent G, Alemu FM. Factors contributing to, and effects of, teenage pregnancy in Juba. South Sudan Medical Journal. 2016; 9(2): 28-31.

10) Hudson DB, Campbell-Grossman C, Kupzyk KA, Brown SE, Yates BC, Hanna KM. Social Support and Psychosocial Well-being Among Low-Income, Adolescent, African American, First-Time Mothers. Clinical Nurse Specialist. 2016; 30(3): 150-8. doi: 10.1097/NUR.0000000000000202. PMID: 27055037.

11) Ngum Chi Watts MC, Liamputtong P, Mcmichael C. Early motherhood: a qualitative study exploring the experiences of African Australian teenage mothers in greater Melbourne, Australia. BMC public health. 2015; 15(1): 1-11. doi: 10.1186/s12889-015-2215-2. PMID: 26358465, PMCID: PMC4566485.

12) Atkinson LD, Peden-McAlpine CJ. Advancing Adolescent Maternal Development: A Grounded Theory. J pediatr nurs. 2014; 29(2): 168-76. doi: 10.1016/j.pedn.2013.08.005. PMID: 24041462.

13) Cook SM, Cameron ST. Social issues of teenage pregnancy. Obstetrics, Gynaecology \& Reproductive Medicine. 2015; 25(9): 243-8. doi: 10.1016/j.ogrm.2015.06.001.

14) Lévesque S, Chamberland C. Resilience, Violence, and Early Pregnancy: A Qualitative Study of the Processes Used by Young Mothers to Overcome Adversities. SAGE Open. 2016; 6(1): 1-15. doi: $10.1177 / 2158244016640851$.

15) Mantovani N, Thomas H. Resilience and survival: Black teenage mothers 'looked after' by the State tell their stories about their experience of care. Children \& Society. 2015; 29(4): 299-309. doi: $10.1111 /$ chso. 12028 .

16) Leerlooijer JN, Bos AE, Ruiter RA, van Reeuwijk MA, Rijsdijk LE, Nshakira N, et al. Qualitative evaluation of the Teenage Mothers Project in Uganda: a community-based empowerment intervention for unmarried teenage mothers. BMC public health. 2013; 13(1): 1. doi: 10.1186/1471-2458-13-816. PMID: 24011141.

17) Aparicio E, Pecukonis EV, O'Neale S. "The love that I was missing": Exploring the lived experience of motherhood among teen mothers in foster care. Children and Youth Services Review. 2015; 51: 44-54. doi: 10.1016/j.childyouth.2015.02.002.

18) Celik R, Törüner EK. The Effect of Technology-Based Breastfeeding Approach on Adolescent Mothers' Breastfeeding Situation. International Journal of Gynecology, Obstetrics and Neonatal Care. 2017; 4(1): 1 6.

19) Logsdon MC, Mittelberg M, Myers J. Use of social media and internet to obtain health information by rural adolescent mothers. Applied Nursing Research. 2015; 28(1): 55-6. PMID: 25015899 doi: 10.1016/j.apnr.2014.04.006.

20) Ehrensaft MK, Knous-Westfall HM, Alonso TL. Web-based prevention of parenting difficulties in young, urban mothers enrolled in post-secondary education. The journal of primary prevention. 2016; 37(6): 52742. doi: 10.1016/j.apnr.2014.04.006. PMID: 25015899.

21) Brand G, Morrison P, Down B. How do health professionals support pregnant and young mothers in the community? A selective review of the research literature. Women and Birth. 2014; 27(3): 174-8. doi: 10.1016/j.wombi.2014.05.004. PMID: 24933525.

22) Freed P, SmithBattle L. Promoting Teen Mothers' Mental Health. MCN: The American Journal of Maternal/Child Nursing. 2016; 41(2): 84-9. doi: 10.1097/NMC.0000000000000216. PMID: 26909721.

23) Sriyasak A, Åkerlind I, Akhavan S. Childrearing among Thai first-time teenage mothers. The Journal of perinatal education. 2013; 22(4): 201. doi: 10.1891/1058-1243.22.4.201. PMID: 24868133.

24) Anwar E, Stanistreet D. 'It has not ruined my life; it has made my life better': a qualitative investigation of the experiences and future aspirations of young mothers from the North West of England. Journal of Public Health. 2015; 37(2): 269-76. doi: 10.1093/pubmed/fdu045. PMID: 25002436.

25) Montazeri S, Gharacheh M, Mohammadi N, Alaghband Rad J, Eftekhar Ardabili H. Determinants of Early Marriage from Married Girls' Perspectives in Iranian Setting: A Qualitative Study. Journal of environmental and public health. 2016; 2016: 1-9. doi: 10.1155/2016/8615929. PMID: 27123012.

26) Dehghan-Nayeri N, Tajvidi M. Experiences of pregnancy among Iranian adolescents: A qualitative study. Iran J Nurs Midwifery Res. 2014; 19(7 Suppl1): S7-12. PMID: 25949255, PMCID: PMC4402980. 
27) Bengtsson M. How to plan and perform a qualitative study using content analysis. Nursing Plus Open. 2016; 2: 8-14. doi: 10.1016/j.npls.2016.01.001.

28) Morse JM. Critical analysis of strategies for determining rigor in qualitative inquiry. Qualitative health research. 2015; 25(9): 1212-22. doi: 10.1177/1049732315588501. PMID: 26184336.

29) Keefe RH, Brownstein-Evans C, Rouland Polmanteer R. "I find peace there": how faith, church, and spirituality help mothers of colour cope with postpartum depression. Mental Health, Religion \& Culture. 2016; 19(7): 722-33. doi: 10.1080/13674676.2016.1244663.

30) Jones R. Giving Voice: The Contested Sites of Motherhood, Religion and Spirituality. Religion \& Gender. 2016; 6(1): 112-7. doi: 10.18352/rg.10129.

31) Logsdon MC, Davis DW, Stikes R, Ratterman R, Ryan L, Myers J. Acceptability and initial efficacy of education for teen mothers. MCN Am J Matern Child Nurs. 2015; 40(3): 186-92. doi: 10.1097/NMC.0000000000000126. PMID: 25919212.

32) Schrag A, Schmidt-Tieszen A. Social support networks of single young mothers. Child and Adolescent Social Work Journal. 2014; 31(4): 315-27. doi: 10.1007/s10560-013-0324-2.

33) de Lima Santos A, Teston EF, Pollyanna Mantelo Cecílio H, Serafim D, Marcon SS. grandmothers involvement in the care of children of adolescent mothers. Rev Min Enferm. 2015; 19(1): 60-4. doi: $10.5935 / 1415-2762.20150005$.

34) de Alencar Lima TNF, Coviello DM, de Alencar Lima MNF, Alves ÉSRC, Davim RMB, Bousquat AEM. Social support networks for adolescent mothers. Revista de enfermagem. 2016; 10(6): 4741-50. doi: 10.5205/reuol.8200-71830-3-SM.1006sup201605.

35) Reibel T, Wyndow P, Walker R. From Consultation to Application: Practical Solutions for Improving Maternal and Neonatal Outcomes for Adolescent Aboriginal Mothers at a Local Level. Healthcare. 2016; 4(4): pii: E90. doi: 10.3390/healthcare4040090. PMID: 27929416, PMCID: PMC5198132. 